\title{
Parameter identification of underwater glider based on particle swarm optimization
}

\author{
Li-Ming Wang \\ Faculty of physics and electronic information, Langfang Teachers University, Langfang \\ 065000, P. R. China \\ E-mail:wlm_shooker@163.com \\ www. lfsf $x y . e d u$
}

\begin{abstract}
An accurate model for an underwater glider is important for the design of a highperformance underwater glider control system. The performance of such control systems is influenced by the parameter variation of underwater glider under real operation conditions. In this paper, the mass parameters of an underwater glider are identified by a particle swarm optimization (PSO) method based on experimental tests. The advantages of adopting the PSO algorithm in this research include easy implementation, high computational efficiency and stable convergence characteristics.
\end{abstract}

Keywords: Underwater Glider; Dynamic Model; Particle Swarm Optimization; UnderActuated System.

\section{Introduction}

In the past few years, an underwater glider (UG) has been widely used in the ocean-graphic surveying, the marine-environment monitoring, the unenclosed aquaculture and ocean exploration, especially in the long-term, large-scale oceanographic monitoring [1]. The UG has a number of attractive features, such as a large range, a high efficiency, a low noise, lower pollution, lower maintenance cost and a compact structure, etc.

Generally, the performance of a UG control system is dependent on the good knowledge of system parameters [2]. The accuracy of a UG model is determined by its parameters, which are the mass and damping coefficient. These parameters should be identified as accurate as possible. There are many analytical methods, which have been proposed for calculating the parameters of a UG [3, 4]. However, most of these methods are based on physical specifications of UG such as the UG mechanical dynamics and the ocean current characteristics. As most of the information is unknown, these analytical methods are not feasible for calculating optimized parameters. Thus, many parameter 
identification methods have been developed such as a neural network system identification method [5], a fuzzy logic system identification method [6] and a system identification intelligent algorithm [7].

Among the system identification intelligent algorithms, particle swarm optimization (PSO) approach [8] is a kind of popular evolutionary computations. Due to the characters of global convergence and fast convergence, nowadays the PSO algorithm is still used as a new and attractive optimization tool and is applied successfully in variety of different fields. For example, Chander et.al [9] presented a new variant of Particle Swarm Optimization (PSO) for image segmentation using optimal multi-level thresholding. In [10], a new approach was introduced for training the adaptive network based fuzzy inference system. In [11], a novel design method for determining the optimal proportionalintegral-derivative controller parameters of an AVR system using the particle swarm optimization algorithm was presented. However, as far as we know, few reports are related to the parameter identification of underwater glider by using particle swarm optimization. Therefore, a PSO algorithm for the parameter identification of underwater glider is meaningful and valuable.

Inspired by the above-mentioned work, in this paper, we investigate the parameter identification of an underwater glider. Consequently, the values of the unknown parameters are identified with the minimum errors. The rest of this paper is organized as follows. A UG model is represented in Section II and its mass parameters are identified by using a PSO algorithm in Section III. Finally in Section VI, a conclusion is given.

\section{Dynamics Equation of Underwater Glide}

The dynamical equation of UV can be described as

$$
\left\{\begin{array}{l}
m_{11} \dot{u}=m_{22} v r-X_{u} u-X_{u|u|} u|u|+F_{u} \\
m_{22} \dot{v}=-m_{11} u r-Y_{v} v-Y_{v|v|} v|v|+F_{r} \\
m_{33} \dot{r}=\left(m_{11}-m_{22}\right) u v-N_{r} r-N_{r|r|} r|r|+F_{r}
\end{array}\right.
$$

Where $m_{11}=m-X_{u}, m_{22}=m-Y_{v}, m_{33}=I_{z}-N_{r}$ It can be found that there is the same input in Eq.(1-B) and Eq.(1-C), thus the system (1) is an underactuated system. 
In this paper, the values of parameters in the system (1) are respectively set as $X_{u}=70 \mathrm{~kg} / \mathrm{s}, X_{u|u|}=100 \mathrm{~kg} / \mathrm{m}, Y_{v}=100 \mathrm{~kg} / \mathrm{s}, Y_{v|v|}=200 \mathrm{~kg} / \mathrm{m}, \quad N_{r}=50 \mathrm{kgm}^{2} / \mathrm{s}$, $N_{r|r|}=100 \mathrm{kgm}^{2}$, so $m_{11}=215 \mathrm{~kg}, m_{22}=265 \mathrm{~kg}, m_{33}=80 \mathrm{kgm}^{2}$.

In the following subsections, the values of $m_{11}, m_{22}, m_{33}$ are assumed to be unknown in advance. Hence, in order to control the behaviors of UG, the values of $m_{11}, m_{22}, m_{33}$ should be identified first. In this paper, we will identify the values of $m_{11}, m_{22}, m_{33}$ by using the particle swarm optimization algorithm.

\section{Parameter Identification of UG}

To identify the unknown parameters $m_{11}, m_{22}, m_{33}$ in the underwater glider by using the particle swarm optimization algorithm, the Eq.(1) is rewritten as

$$
\begin{aligned}
& \left(\begin{array}{c}
\dot{u} \\
\dot{v} \\
\dot{r}
\end{array}\right)+\left(\begin{array}{ccc}
\frac{X_{u}}{m_{11}} & 0 & 0 \\
0 & \frac{Y_{v}}{m_{22}} & 0 \\
0 & 0 & \frac{N_{r}}{m_{33}}
\end{array}\right)\left(\begin{array}{l}
u \\
v \\
r
\end{array}\right)+\left(\begin{array}{l}
\frac{X_{u|u|}}{m_{11}} u|u|-\frac{m_{22}}{m_{11}} v r \\
\frac{Y_{v|v|}}{m_{22}} v|v|+\frac{m_{11}}{m_{22}} u r \\
\frac{N_{r|r|} r|r|}{m_{33}}-\frac{m_{11}-m_{22}}{m_{33}} u v
\end{array}\right)=\left(\begin{array}{cc}
\frac{1}{m_{11}} & 0 \\
0 & \frac{1}{m_{22}} \\
0 & \frac{1}{m_{33}}
\end{array}\right)\left(\begin{array}{l}
F_{u} \\
F_{r}
\end{array}\right) \\
& \text { Let } Y=\left(\begin{array}{c}
\dot{u} \\
\dot{v} \\
\dot{r}
\end{array}\right)+\left(\begin{array}{ccc}
\frac{X_{u}}{m_{11}} & 0 & 0 \\
0 & \frac{Y_{v}}{m_{22}} & 0 \\
0 & 0 & \frac{N_{r}}{m_{33}}
\end{array}\right)\left(\begin{array}{l}
u \\
v \\
r
\end{array}\right)+\left(\begin{array}{l}
\frac{X_{u|u|}}{m_{11}} u|u|-\frac{m_{22}}{m_{11}} v r \\
\frac{Y_{v|v|}}{m_{22}} v|v|+\frac{m_{11}}{m_{22}} u r \\
\frac{N_{r|r|} r|r|}{m_{33}}-\frac{m_{11}-m_{22}}{m_{33}} u v
\end{array}\right) \\
& \text { and } A=\left(\begin{array}{cc}
\frac{1}{m_{11}} & 0 \\
0 & \frac{1}{m_{22}} \\
0 & \frac{1}{m_{33}}
\end{array}\right) \text { and } \tau=\left(\begin{array}{c}
F_{u} \\
F_{r}
\end{array}\right) \text {. }
\end{aligned}
$$

The Eq.(2) can be further rewritten as

$$
Y=A \tau
$$


where $Y(1)=\frac{1}{m_{11}} F_{u}, Y(2)=\frac{1}{m_{22}} F_{r}, Y(3)=\frac{1}{m_{33}} F_{r}$.

Since $\frac{1}{m_{11}}, \frac{1}{m_{22}}$ and $\frac{1}{m_{33}}$ are linearly independent, we can identify the values of $m_{11}, m_{22}, m_{33}$ by using the particle swarm optimization algorithm when $\tau$ and $Y$ are respectively regarded as input and output and can be observed.

When using the PSO algorithm for parameters identification, the fitness function is defined as $J=\frac{1}{2} \sum_{i=1}^{N}\left(y_{i}-\hat{y}_{i}\right)^{T}\left(y_{i}-\hat{y}_{i}\right)$. The basic steps of parameters identification based on the PSO algorithm are as follows:

1) Initializing population and setting parameters;

2) Sampling the actual inputs and outputs of the system;

3) Calculating the fitness value for each particle;

4) Updating the position and the speed of particles using the following equations $\left\{\begin{array}{l}v_{i}^{n+1}=w v_{i}^{n}+c_{1} r_{1}\left(p_{i}^{n}-x_{i}^{n}\right)+c_{2} r_{2}\left(p_{i}^{n}-x_{i}^{n}\right) \\ x_{i}^{n+1}=x_{i}^{n}+v_{i}^{n+1}\end{array}\right.$

5) Repeating steps 3) and 4) until stopping criteria are met.

There are three parameters $m_{11}, m_{22}, m_{33}$ to be identified in this system. In the simulation, we set the identified parameter as $\hat{P}=\left[\hat{m}_{11}, \hat{m}_{22}, \hat{m}_{33}\right]$ and its actual parameter as $P=\left[m_{11}, m_{22}, m_{33}\right]=\left[215 \mathrm{~kg}, 265 \mathrm{~kg}, 80 \mathrm{kgm}^{2}\right]$. Therefore, we set $m_{11} \in[0,300], m_{22} \in[0,300]$ and $m_{33} \in[0,100]$. The weight PSO algorithm ${ }^{[8]}$ is applied to identify the above-mentioned unknown parameters. In this algorithm, the swarm population size is set as $N=60$ and $c_{1}=c_{2}=1.49$. The constriction factor $w$ is set as $w=0.82 \cdot v_{\min }=0$ and $v_{\max }=20$ are set to be the lower and upper bounds of allowed speed ranges, respectively. In order to assure an appropriate accuracy, the final results are averaged over up to 100 independent runs. 

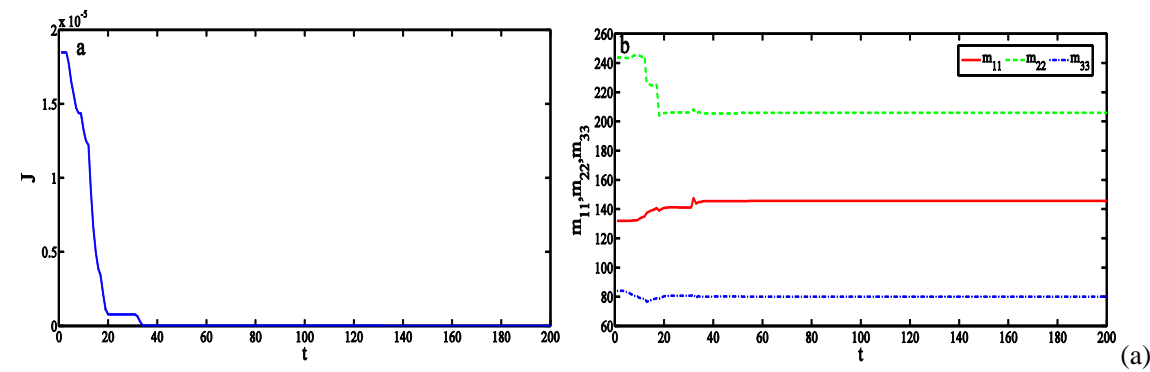

The optimization process of identification error function $J$ (b) The identification processes of unknown parameters $m_{11}, m_{22}, m_{33}$

Fig. 1 Numerical simulation results

To study the convergence speed of the propose parameter identification, the convergence processes of the fitness function is visualized in Fig.1. Fig.1(a) shows that the PSO algorithm finds the global optima in less than 100 iterations. Fig.1(b) further shows the identification processes of parameters by using the PSO algorithm. From Fig.1 we can find that the identification value of each parameter can be relatively fast return to the vicinity of its true value, which indicates that the proposed PSO-base parameter identification algorithm can relatively accurately identify the value of unknown parameter.

\section{Conclusion}

In this paper, based on the particle swarm optimization approach, the unknown parameters in an underwater glider are accurately. The simulation results show the effectiveness of the proposed method. It should be noted that the parameters identification is a very important step in the process of design and development for the underwater glider. Therefore, the results of this paper are more applicable and more representative. We hope our works can contribute to the parameters identification and the behaviors control for an underwater glider. As for the database fault diagnosis for an underwater glider, we will discuss elsewhere.

\section{Acknowledgments}

This work was partially supported by the Projects of Department of Science and Technology in Hebei Province, China (Grant No.15213401), the Research Foundation of Education Bureau of Hebei Province, China (Grant No.QN2014096) and the Langfang Teachers University Foundation, China (Grant no. LSLZ201404). 


\section{References}

1. F. E. Farris and W. M. Rand, "Underwater glider (Patent style)," U.S. Patent 3157 145, November 17, 1964.

2. J. G. Graver, N. E. Leonard, "Underwater glider dynamics and control," 12th international symposium on unmanned untethered submersible technology, 2001, pp. 1742-1710.

3. A. Bender, D. M. Steinberg, A. L. Friedman, et al, "Analysis of an autonomous underwater glider," Proceedings of the Australasian Conference on Robotics and Automation, 2008, pp. 1-10.

4. R. Grasso, D. Cecchi, M. Cococcioni, et al, "Model based decision support for underwater glider operation monitoring," Oceans 2010. IEEE, 2010, pp.1-8.

5. K. S. Narendra, K. Parthasarathy. Identification and control of dynamical systems using neural networks," Neural Networks, IEEE Transactions on, vol. 1, no. 1, pp. 4-27, January 1990.

6. T. Takagi, M. Sugeno, "Fuzzy identification of systems and its applications to modeling and control," Systems, Man and Cybernetics, IEEE Transactions on, vol. 12, no. 1, pp. 116-132,April 1985.

7. K. Kristinsson, G. A. Dumont, "System identification and control using genetic algorithms," Systems, Man and Cybernetics, IEEE Transactions on, vol. 22, no. 5, pp. 1033-1046, May 1992.

8. J. Kennedy, "Particle swarm optimization," US: Springer, 2011.

9. A. Chander, A. Chatterjee, P. Siarry, "A new social and momentum component adaptive PSO algorithm for image segmentation," Expert Systems with Applications, vol. 38, no. 5, pp. 4998-5004, May 2011.

10. V. S. Ghomsheh, M. A. Shoorehdeli, M. Teshnehlab, "Training ANFIS structure with modified PSO algorithm," Control \& Automation, 2007. MED'07. Mediterranean Conference on. IEEE, 2007, pp.1-6.

11. Z. L. Gaing, "A particle swarm optimization approach for optimum design of PID controller in AVR system," Energy Conversion, IEEE Transactions on, vol. 19, no. 2, pp. 384-391, February 2004. 